\title{
Conduction and Resistive Switching Memory in Al/MoS2+PVP/Ag Devices Fabricated using Drop cast Method
}

Zolile Wiseman Dlamini ( $D$ 46118357@mylife.unisa.ac.za )

UNIVERSITY OF SOUTH AFRICA

Sreedevi Vallabhapurapu

University of South Africa

Tebogo Sfiso Mahule

University of South Africa

Shuying Wu

Macquarie University

Vijaya Srinivasu Vallabhapurapu

University of South Africa

Research Article

Keywords: MoS2, PVP, Resistive switching, drop cast

Posted Date: October 6th, 2021

DOI: https://doi.org/10.21203/rs.3.rs-956321/v1

License: (9) This work is licensed under a Creative Commons Attribution 4.0 International License.

Read Full License 


\title{
Conduction and Resistive Switching Memory in Al/MoS $2+P V P / A g$ Devices Fabri- cated using Drop cast Method
}

\author{
Zolile Wiseman Dlamini ${ }^{1,2,}{ }^{*}$, Sreedevi Vallabhapurapu ${ }^{3}$, Tebogo Sfiso Mahule ${ }^{1}$, Shuying $\mathrm{Wu}^{4}$ and \\ Vijaya Srinivasu Vallabhapurapu ${ }^{1}$ \\ ${ }^{1}$ Department of Physics, University of South Africa, 28 Pioneer Ave, Florida Park, 1709, Gauteng, South Af- \\ rica \\ ${ }^{2}$ Department of Maths, Science and Technology Education, Central University of Technology, 20 President \\ Brand St., Bloemfontein, 9300, Free State, South Africa. \\ ${ }^{3}$ School of Computing, University of South Africa, 28 Pioneer Ave, Florida Park, 1709, Gauteng, South Af- \\ rica. \\ ${ }^{4}$ School of Engineering, Macquarie University, Balaclava Road, Macquarie Park NSW, 2109, Sydney, Aus- \\ tralia. \\ ${ }^{*}$ Corresponding author. E-Mail: zoliledlamini@hotmail.com
}

\begin{abstract}
Resistive switching in $\mathrm{MoS}_{2}$ embedded PVP composite-based ReRAM with Al and Ag electrodes is reported. A cost-free drop cast method was used to deposit active layers consisting of $30 \mathrm{wt} \%, 40 \mathrm{wt} \%$, and 70 wt\% of $\mathrm{MoS}_{2}$ in PVP. Each system exhibited unique electroforming and switching mode. Asymmetrical bipolar resistive switching occurring only in the positive voltage bias, a typical bipolar resistive switching and a typical 'O-type' resistive switching were observed for the $30 \mathrm{wt} \%$, $40 \mathrm{wt} \%$, and $70 \mathrm{wt} \%$ systems, respectively. Furthermore, injection of charge carriers at the electrode/active layer interface and electrochemical metalization mechanisms drove the formation of a nanoscale conductive filament in the device A and B. On the other hand, we attributed the conduction mechanism of device $C$ to hopping conduction. Our results demonstrate the behaviour of $\mathrm{MoS}_{2}$ embedded PVP composite-based ReRAM has a strong dependence on the amount of $\mathrm{MoS}_{2}$ and that both the switching and conduction mechanism can be exploited by controlling the amount of $\mathrm{MoS}_{2}$ in the composite.
\end{abstract}

Keywords: MoS2, PVP, Resistive switching, drop cast

\section{Introduction}

Research efforts are put towards developing biodegradable electronics devices to reduce environmental pollution and toxins caused by the disposal of current electronic devices. The memory device industry also develops next-generation resistive switching memory (ReRAM) using biodegradable materials[1-5]. ReRAM uses a dielectric material called an active layer sandwiched between two electrodes. Each ReRAM cell has two distinctive resistive states, i.e., a high resistive state (HRS) and a low resistive state (LRS), which are selectable using electric stimuli[6]. Since their discovery[7], ReRAMs have shown good properties such as minuscule cell size $(<10 \mathrm{~nm})$, long retention time $\left(>10^{6} \mathrm{~s}\right)$, high operating speed $(\mathrm{sub}-\mathrm{ns})$, multilevel data storage capability, good endurance, large ON/OFF ratio and many more[35], making them a potential replacement of the current memory giants, i.e., dynamic random access memory (DRAM) and Flash memory. Current memory devices seem to not comply with the projected Si-Logic level of up to $0.1 \mathrm{~V}$ operating voltage[8]. Poly(vinylpyrrolidone)(PVP) is a water-soluble and nontoxic synthetic polymer permitted for use in the 
cosmetics, food, and drug industries[9]. PVP blended with other polymers, such as polyacrylamide[10], and other complexes such as $\mathrm{KIO}_{4}[11]$ have demonstrated good electrical properties in thin-film samples. PVP has also shown strong interaction with metal cations[12], making it possible to tailor its electronic properties for various applications such as batteries[13]. PVP dispersed with $\mathrm{MoS}_{2}$ ReRAM systems have shown exciting memory behaviour. Bai et al. [14] observed three switching modes, i.e., bipolar, asymmetric bipolar and threshold switching(TS) in the same system of PVP and $\mathrm{MoS}_{2}$ composite sandwiched between Al and ITO (indium doped tin oxide) electrodes. Wu et al. [15] reported bipolar switching mode and that the energy consumption in $\mathrm{MoS}_{2}$ and PVP device with Pt and ITO electrodes can be decreased by doping $\mathrm{MoS}_{2}$ with nitrogen. Rehman et al. [16] also reported bipolar switching in PVP and $\mathrm{MoS}_{2}$ device with Ag acting as both bottom electrode (BE) and top electrode (TE).

We report on low operating voltage PVP, and $\mathrm{MoS}_{2}$ based ReRAM devices that use Al and $\mathrm{Ag}$ as $\mathrm{BT}$ and TE, respectively. Our study focuses on the effect of $\mathrm{MoS}_{2}$ weight percentage in the PVP/MoS 2 composite on both switching mode and conduction mechanism. Also, our ReRAMs are fabricated using the drop cast method, which does not require any use of electricity.

\section{Experimental details}

\subsection{Materials and Methods}

PVP powder and MoS2 powders were each dissolved in isopropyl alcohol (IPA) to make $2.5 \mathrm{w} / \mathrm{v} \%$ (2.5 g PVP into $100 \mathrm{ml} \mathrm{IPA}$ ) and $1 \mathrm{w} / \mathrm{v} \%$ (1 g MoS2 into $100 \mathrm{ml}$ IPA) solutions, respectively. The solutions were stirred for over 4 hours at room temperature until a homogeneous PVP solution was obtained. The solutions were then mixed to make $30 \mathrm{wt} \%, 40 \mathrm{wt} \%$, and $70 \mathrm{wt} \%$ MoS2 into PVP.

Al sheet (1.00 mm thick, Sigma Aldrich, Prod. No. 266957) was cut into pieces and precleaned by sonication ( $5 \mathrm{~min}$ in acetone, $5 \mathrm{~min}$ in isopropyl alcohol, and $5 \mathrm{~min}$ in ultra-distilled water, in that order). The active layers ( $\approx 7-10 \mu \mathrm{m}$ thick) were deposited by dropping few drops of PVP+MoS 2 composite with $30 \mathrm{wt} \%$ (Device A), $40 \mathrm{wt} \%$ (Device B), and $70 \mathrm{wt} \%$ (Device C) on Al substrates. The active layers were dried at room temperature for 48 hours. Ag paste (Sigma Aldrich, Prod. No. 735825) was applied on the active layer as top electrodes. For electrical characterization, a Precision Source/Measure Unit (SMU, Keysight B2901A) was sued to study the current-voltage behaviour of our devices. Current-voltage measurements were performed at room temperature by applying voltage stress at the top electrode while the bottom electrode was grounded. This voltage was increased from zero until a sudden increase in current indicating electroforming was observed. After electroforming, the voltage was swept from $0 \rightarrow V_{M A X} \rightarrow 0 \rightarrow-V_{M A X} \rightarrow 0$ study the memory behaviour.

\section{Results and discussion}

Figure 1 shows the results of the electric study of all the studied devices. The electroforming I-V characteristics of device $A$ is shown in 
Figure 1(a). This graph reveals no current in the $0-1.0 \mathrm{~V}$ regime. When the voltage exceeds $1.0 \mathrm{~V}$, a gradual increase in current with voltage was observed, which became more visible beyond $1.78 \mathrm{~V}$. When the voltage exceeds $2 \mathrm{~V}$, a steep rise in current takes place, leading to an abrupt jump at $2.68 \mathrm{~V}$, indicating that the device is electroforming. For comparison, we shall look at the electroforming behaviour of devices $B$ and $C$, shown in

Figure 1 (c and e), respectively. For device $B$, the graph reveals linear current variation with a relatively high slope $\left(\right.$ Slope $=3.22 \times 10^{-6} \Omega^{-1}$ ) in the region $0-1.05 \mathrm{~V}$, followed by an abrupt jump at $1.05 \mathrm{~V}$, indicating electroforming. Device $\mathrm{C}^{\prime}$ 's graph, on the other hand, depicts a weak current variation with applied voltage (Slope $\left.=1.46 \times 10^{-7} \Omega^{-1}\right)$ in the $0.5 \mathrm{~V}-$ $1.30 \mathrm{~V}$ region, after which an increase (Slope $=3.41 \times 10^{-6} \Omega^{-1}$ ) in slope is observed, followed by electroforming at $1.70 \mathrm{~V}$. Our results show that these three systems have different electroforming behaviour due to varying amounts of $\mathrm{MoS}_{2}$ content on each system. Next, we look at the semilogarithmic I-V characteristics of these devices to understand their memory behaviour.

Figure 1(b) shows the I-V features of device A. For this device, an increase in applied voltage in the positive bias increased the current until a jump from a low current, i.e., high resistive state (HRS) to high current, i.e., low resistive state (LRS), occurs at 1.30 V. The HRS-to-LRS switch is called the SET process. Upon decreasing the voltage, the device remains in the LRS, as the current did not retrace its previous path. Thus creating an I-V hysteresis, which indicates memory behaviour. For this device, a fair $\left(>10^{2}\right)$ ON/OFF ratio is achieved. In the negative voltage bias, the down and upsweeps currents almost coincide and show very weak hysteresis. This indicates that the switching in this device is only in the positive voltage bias. Such memory behaviour has been reported before in $\mathrm{ZnO} / \alpha-\mathrm{Fe}_{2} \mathrm{O}_{3}$ core-shell based nano-rods[17]. For device $\mathrm{B}$, the semilogarithmic I-V characteristic graph is shown in

Figure 1(d). For this device, a low voltage switching, with $V_{S E T}=+0.60 \mathrm{~V}$, is observed in the positive voltage bias, and the $V_{R E S E T}$ process is not shown in the graph. However, a small hysteresis in the negative voltage bias is also present.

Figure $1(\mathrm{f})$ shows the semilogarithmic I-V characteristics of device $\mathrm{C}$. For this device, even though there are some minor jumps in current, the semilogarithmic I-V hysteresis resembles the 'O-type' memory hysteresis. 'O-type' resistive switching is characterized by homogeneous gradually increasing or decreasing current such that the complete cycle curve corresponds to a letter ' $\mathrm{O}$ '[18]. These devices show different electroforming and memory behaviour attributed to different $\mathrm{MoS}_{2}$ weight percentages in the active layer. In the following subsection, we shall analyze the conduction mechanism on each device. 

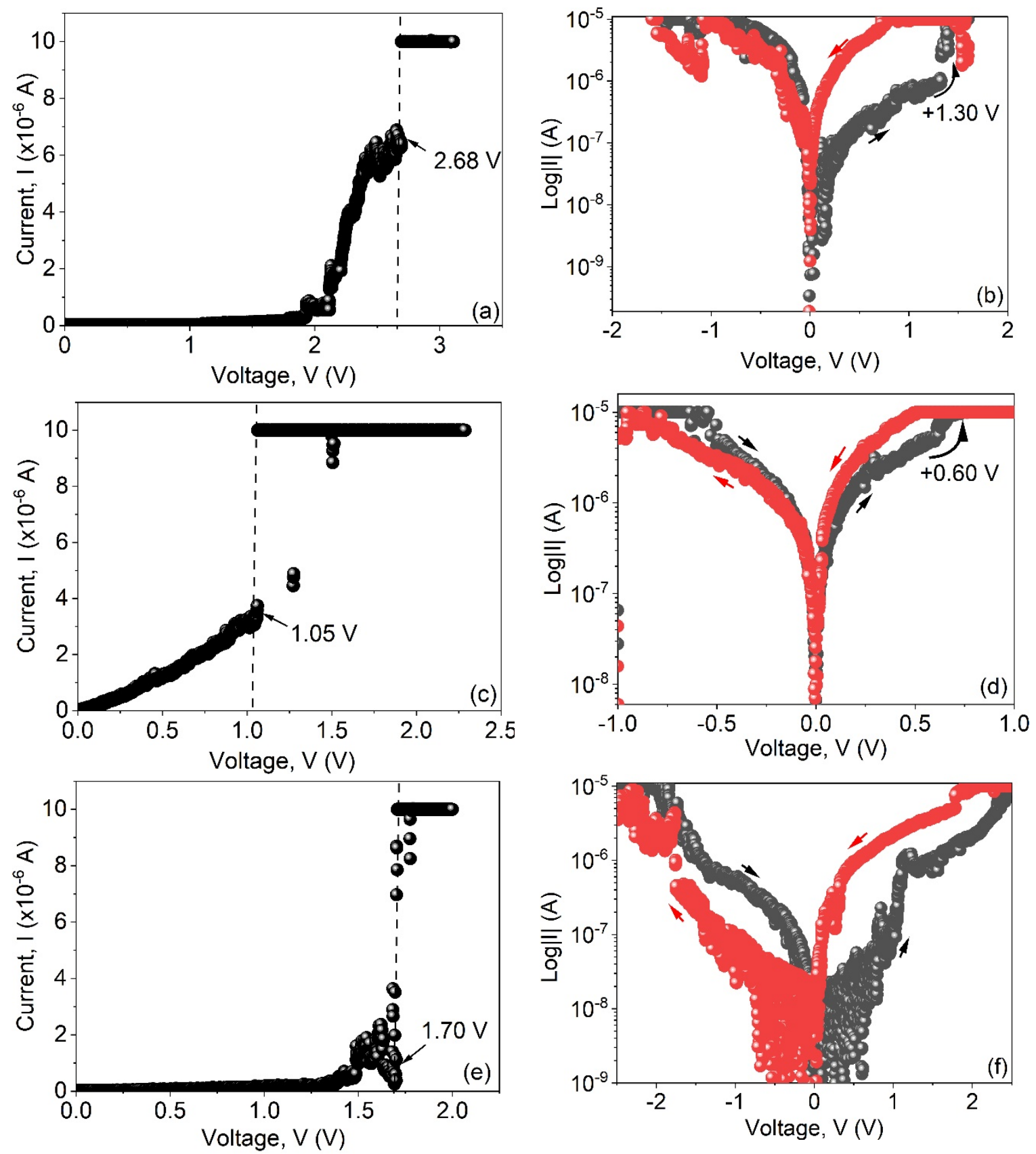

Figure 1. Current-voltage characteristics graphs depicting the (a) electroforming curve and (b) memory behaviour of device $A$; (c) electroforming curve and (d) memory behaviour of device $B$; and (e) electroforming and memory behaviour of device $C$.

\subsection{Conduction mechanism}

When voltage stress, $\mathrm{V}$ is applied at one of the electrodes while the other electrode is grounded, a current density, J may pass in the dielectric such that, $J \propto V^{n}$ [19]. The value of 
$n$ associates with the dominant conduction mechanism and can be determined by plotting I-V data on a log-log scale. The review article by Chiu[20] provides a summary of the already proposed mechanisms. Figure 2(a) shows the log-log graph of device A. For this device, the curve fitting of the ON-state current shows $n=1.33 \sim 1$, which implies Ohmic behaviour. The Ohmic behaviour indicates that thermally activated charge carriers (free electrons) are responsible for the conduction, and the current is directly proportional to the applied electric field[21-23]. The current density obeying Ohm's Law is given as[22]:

$$
J_{O h m}=q \mu N_{C} E \exp \left[\frac{-\left(E_{C}-E_{F}\right)}{k T}\right]
$$

Here $\mu$ is the mobility of the charge carriers, $N_{C}$ is the density of state of the conduction band, $E_{C}$ is the energy of the conduction band, $E_{F}$ is the Fermi energy, $\mathrm{T}$ is the temperature in Kelvin and $k$ is the Boltzmann constant. Ohms law indicates typical metallic conduction due to the formation of nanoscale conduction filaments (CFs) through the active layer. CF switching has been reported in many ReRAM systems. However, different mechanisms can lead to the formation of CFs; therefore, we must analyze the OFF-state current behaviour. The OFF-state current fit shows $n=1.59$. This obtained value of $n$ rules out both Ohmic conduction and the space-charge limited conduction (SCLC) mechanism, where $n=2$ [24]. This is still an uncharacterized conduction mechanism. However, according to Perera et al. [24], this current variation characteristic indicates that the ejection of carriers limits the current at the interface.

The log-log I-V data fitting device B is shown in Figure 2(b). Our data shows that $n=1$ in both the ON- and OFF- state. The occurrence of Ohmic conduction in both LRS and HRS is is associated with a typical electrochemical metalization (ECM) conduction mechanism, where CFs are formed through the reduction $\left(M^{n+}+n e^{-} \rightarrow M\right)$ of metal ions $[24,25]$. Our systems consist of an electrochemically active $\mathrm{Ag}$ electrode, but because the ECM behaviour is observed only in device $\mathrm{B}$, this may suggest that the CFs are formed through the reduction process of metal ions other than $\mathrm{Ag}^{+}$ions. In comparison, more $\mathrm{MoS}_{2}$ particles are in device $B$ than in device $A$. Therefore, we believe that the reduction of $\mathrm{Mo}^{4+}$ ions domination is more possible in device $\mathrm{B}$ than in device $\mathrm{A}$. Which explains the difference in $n$ values for these two devices.

Figure 3(c) shows the log-log fitting of device C. For this device $n=1.5$ for the ON-state, while 4 for the OFF-state. Again, $n=4$ implies a strong power relationship, current-voltage relationship. We fitted this OFF-state data with different functions and found that the exponential function fits the data perfectly, as shown in Fig. 3(b). The fitted exponential function corresponds perfectly with the hopping conduction mechanism expression given as[26]:

$$
I=I_{0}+A \exp \left[\frac{V-V_{a}}{k T}\right]
$$

Here, $I_{0}$ is the residual current, $\mathrm{A}$ is a constant, $V_{a}$ is a characteristic voltage, $k$ is the Boltzmann constant, and $T$ is the temperature in Kelvin[26]. These results may imply that a large amount of $\mathrm{MoS}_{2}$ content in the PVP medium provides room for electrons to hop from the electrode to the MoS $2, \mathrm{MoS}_{2}$ to MoS 2 and lastly MoS 2 to the other electrode. 

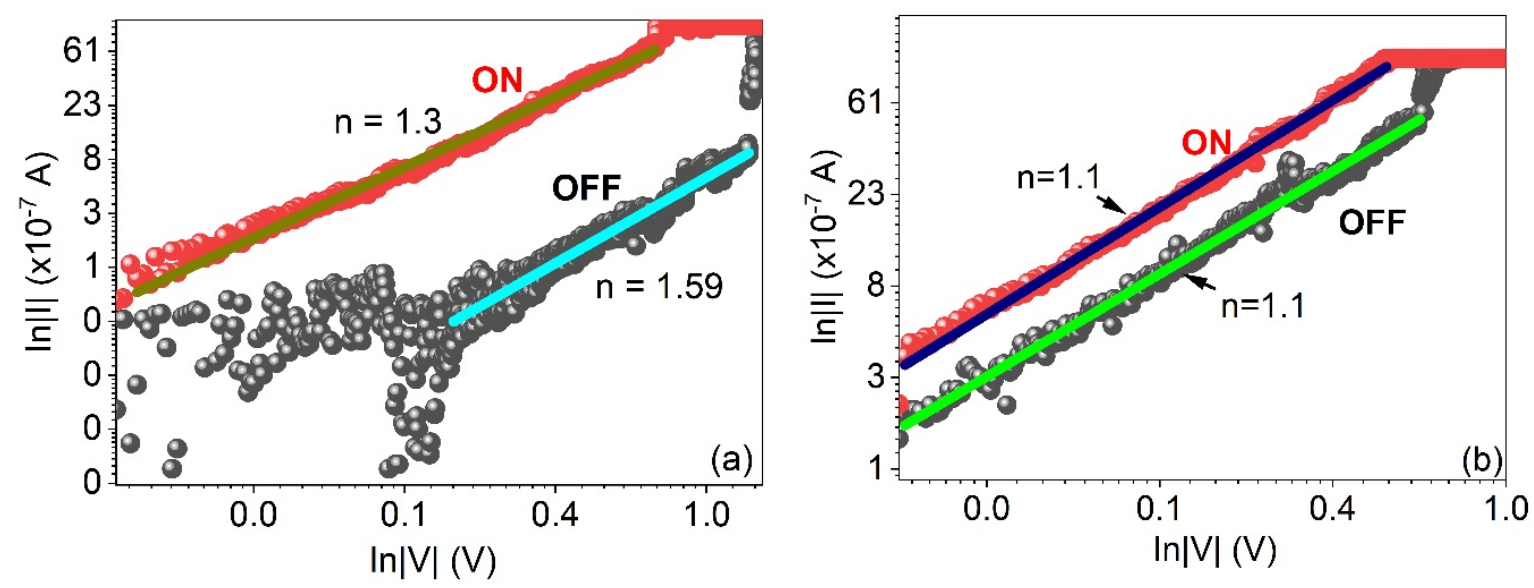

Figure 2. $\ln I-\ln V$ curve fitting of (a) Device A and (b) Device B.
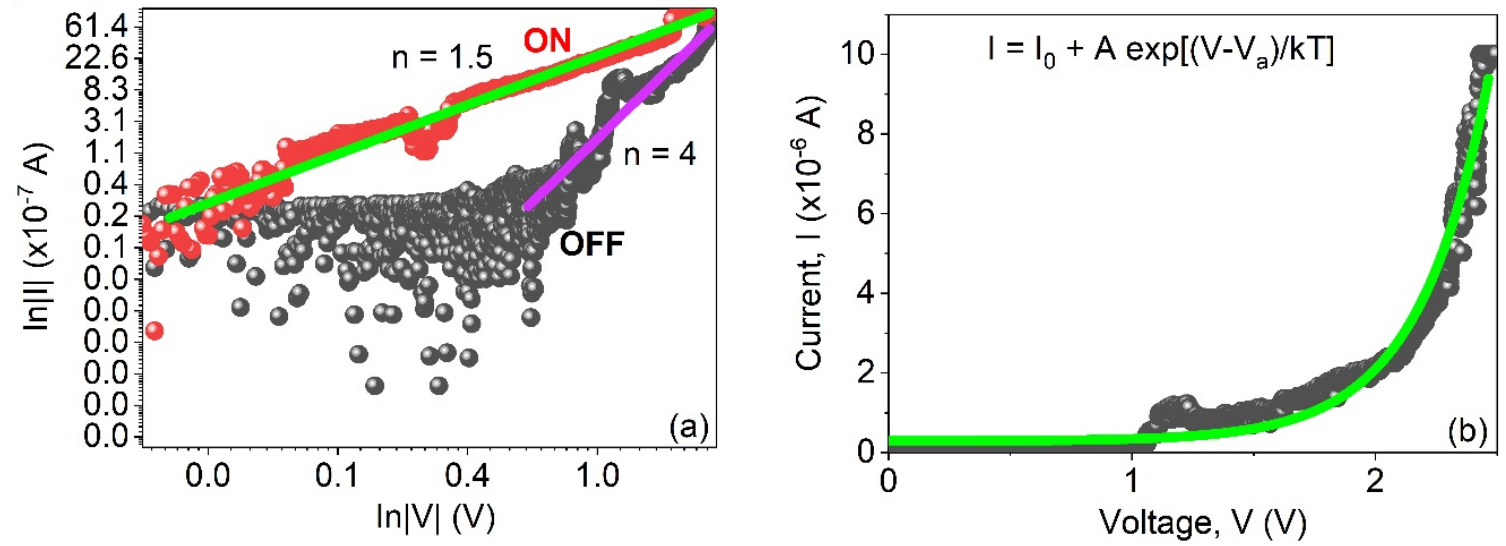

Figure 3. (a) In $I-\ln V$ curve fitting and (b) the corresponging exponential curve fitting of the OFF-state current of Device $C$.

\section{Conclusion}

In summary, we have fabricated the Al/PVP+MoS $2 / \mathrm{Ag}$ ReRAM using the cost-free drop cast method. Memory behaviour at operating voltage as low as $0.60 \mathrm{~V}$ was achieved in the device with $40 w t \% \mathrm{MoS}_{2}$ to PVP. Low operating voltage allows the device to be utilized as a low power device. As a highlight to our results, we found that devices with different $\mathrm{MoS}_{2}$ content demonstrated different resistive switching modes and conducting mechanisms, which implies that both memory behaviour and switching in the PVP/MoS 2 composite based ReRAMs can be exploited by controlling the amount of $\mathrm{MoS}_{2}$.

\section{Acknowledgements}

Zolile acknowledges the financial support from the DHET-nGAP program provided through Central University of Technology. 


\section{References}

1. N. Raeis-hosseini and J. Lee, 223 (2017).doi:10.1007/s10832-017-0104-z

2. S. Liu, S. Dong, X. Wang, L. Shi, H. Xu, S. Huang, and J. Luo, Nanotechnology 31 (2020).

3. J. Xu, X. Zhao, Z. Wang, H. Xu, J. Hu, J. Ma, and Y. Liu, Small 15, 1803970 (2019).

4. $\quad$ N. R. Hosseini and J. S. Lee, Adv. Funct. Mater. 25, 5586 (2015).

5. S. Vallabhapurapu, L. D. Varma Sangani, M. Ghanashyam Krishna, V. V. Srinivasu, C. Du, S. Du, and A. Srinivasan, Mater. Today Proc. 9, 615 (2019).

6. S. Yu, Synth. Lect. Emerg. Eng. Technol. 2, 1 (2016).

7. T. W. Hickmott, J. Appl. Phys. 33, 2669 (1962).

8. D. S. Jeong, R. Thomas, R. S. Katiyar, J. F. Scott, H. Kohlstedt, A. Petraru, and C. S. Hwang, Reports Prog. Phys. 75, 076502 (2012).

9. B. Nair, Int. J. Toxicol. 17, 95 (1998).

10. A. Rawat, H. K. Mahavar, A. Tanwar, and P. J. Singh, Bull. Mater. Sci. 37, 273 (2014).

11. M. Ravi, S. Bhavani, K. Kiran Kumar, and V. V. R. Narasimaha Rao, Solid State Sci. 19, 85 (2013).

12. M. Liu, X. Yan, H. Liu, and W. Yu, React. Funct. Polym. 44, 55 (2000).

13. C. V. S. Reddy, A. Jin, X. Han, Q. Zhu, L. Mai, and W. Chen, 8, 279 (2006).

14. N. Bai, M. Xu, C. Hu, Y. Ma, Q. Wang, D. He, J. Qi, and Y. Li, Mater. Sci. Semicond. Process. 91, 246 (2019).

15. Z. Wu, T. Wang, C. Sun, P. Liu, B. Xia, J. Zhang, Y. Liu, and D. Gao, 125213 (2017).

16. M. M. Rehman, G. U. Siddiqui, J. Z. Gul, S. W. Kim, J. H. Lim, and K. H. Choi, Sci. Rep. 6, 1 (2016).

17. D. Sarkar and A. K. Singh, J. Phys. Chem. C 121, 12953 (2017).

18. D. Prime and S. Paul, Philos. Trans. R. Soc. A Math. Phys. Eng. Sci. 367, 4141 (2009).

19. Y. Liu, P. Gao, X. Jiang, L. Li, J. Zhang, and W. Peng, J. Appl. Phys. 116 (2014).

20. F. Chiu, Adv. Mater. Sci. Eng. 2014, 1 (2014).

21. E. Lim and R. Ismail, Electronics 4, 586 (2015).

22. S. Qin, R. Dong, X. Yan, and Q. Du, Org. Electron. 22, 147 (2015).

23. R. Perera, A. Ikeda, R. Hattori, and Y. Kuroki, Microelectron. Eng. 65, 357 (2003).

24. Z. Shen, C. Zhao, Y. Qi, W. Xu, Y. Liu, I. Z. Mitrovic, L. Yang, and C. Zhao, Nanomaterials 10, 1 (2020).

25. N. Raeis-Hosseini, S. Lim, H. Hwang, and J. Rho, Adv. Electron. Mater. 4 (2018).

26. S. Vallabhapurapu, A. Rohom, N. B. Chaure, C. Tu, S. Du, V. V. Srinivasu, and A. Srinivasan, Appl. Phys. A 124, 639 (2018). 